\title{
Time-Domain Homogenization of Foil Windings in 2-D Magnetodynamic Finite-Element Models
}

\author{
Carlos A. Valdivieso ${ }^{1,2,4}$, Brahim Ramdane ${ }^{1}$, Gerard Meunier ${ }^{1}$, Ruth V. Sabariego ${ }^{2}$, Johan Gyselinck ${ }^{3}$ and \\ Christophe Guerin ${ }^{4}$ \\ ${ }^{1}$ Univ. Grenoble Alpes, CNRS, Grenoble INP, G2Elab, F-38000 Grenoble, France \\ ${ }^{2} \mathrm{KU}$ Leuven, Dept. Electrical Engineering, EnergyVille, 3000 Leuven, Belgium \\ ${ }^{3}$ Université libre de Bruxelles, BEAMS department, 1050 Brussels, Belgium \\ ${ }^{4}$ Altair Engineering France, 38240 Meylan, France
}

\begin{abstract}
In this paper, an approach for the time-domain homogenization of foil windings in two-dimensional (2-D) finite-element (FE) models is presented. The homogenized formulation is characterized by an axial current redistribution and a radial interturn voltage gradient. The method is successfully applied to an axisymmetric 18-turn foil-winding inductor. The local and global results agree very well with those obtained by an accurate but expensive FE model in which all turns are explicitly discretized.
\end{abstract}

Index Terms—Eddy currents, finite element method, foil windings, proximity effect, skin effect, time domain.

\section{INTRODUCTION}

$\mathbf{F}$ OIL windings are extensively used in transformers and inductors in a wide range of frequencies and rated powers. For these devices, skin and proximity effects are essential aspects at the design stage. To this end, some analytical approaches have been proposed in [1], [2]. Numerical methods can also be used, in particular the FE method. Classically, the eddy-current analysis for foil windings in FE models requires the representation of each separate turn of the winding, which leads to computationally expensive simulations with a prohibitive number of unknowns [3]. Dedicated homogenization techniques for foil windings so far available in literature are limited to the frequency domain. Time domain analysis has an important role as well, it allows the representation of transient phenomena (such as e.g., inrush currents), the operation under non-sinusoidal excitations or non-linearity. In this paper, a time-domain extension of the developments done in [4], [5] is proposed.

\section{Magnetodynamic a- $v$ Formulation}

A bounded domain $\Gamma$ of the 2D Euclidean space is considered. The conducting and nonconducting subdomains are denoted $\Gamma_{c}$ and $\Gamma_{n c}$ respectively, with $\Gamma=\Gamma_{c} \cup \Gamma_{n c}$. For eddycurrent problems, the electric field $\mathbf{e}$ is expressed in terms of a magnetic vector potential $\mathbf{a}$ and an electric scalar potential $v$ as

$$
\mathbf{e}=-\frac{\partial \mathbf{a}}{\partial t}-\operatorname{grad} v \quad \text { with } \quad \mathbf{b}=\operatorname{curl} \mathbf{a},
$$

where $\mathbf{b}$ is the magnetic flux density.

A typical foil winding of cylindrical disposition with $N_{f}$ turns, axial height $h_{z}$ and total radial width $h_{r}$ is considered and presented in Fig. $1 \mathrm{a}$ In cylindrical coordinates $(r, \phi, z)$, a can be represented as a scalar field $\mathrm{a}_{\phi}$ and $\operatorname{grad} v$ as a voltage drop $U$, both along the $\phi$-direction. The current density can be expressed as

$$
j_{\phi}=-\lambda \sigma \frac{\partial \mathrm{a}_{\phi}}{\partial t}-\lambda \sigma \frac{U}{2 \pi r}
$$

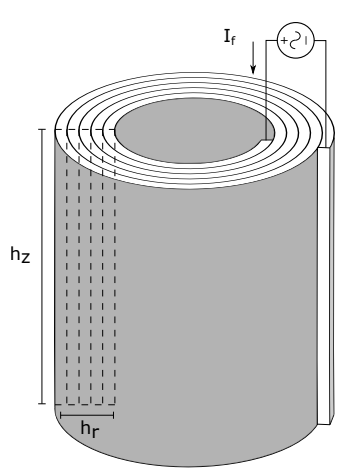

(a)

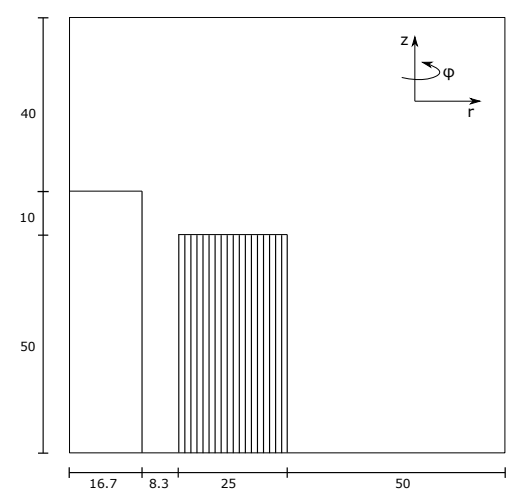

(b)
Fig. 1: Foil winding model: (a) physical representation and (b) test case geometry (upper half, dimensions: $\mathrm{mm}$ ).

where $\sigma$ is the conductivity and $\lambda$ the fill factor. For low frequency devices operating at rated conditions, the skin effect is predominant in the axial direction, since the width of the foil is usually smaller than the skin depth. Hence, the current density can be assumed constant in the radial direction [5]. For a single foil turn we have

$$
\frac{h_{r}}{N_{f}} \int_{h_{z}} j_{\phi} d z=I_{f},
$$

where $I_{f}$ is the foil current. Additionally, a voltage gradient will be present in the axial direction given the in-between insulation layers. Considering that the turns will not be geometrically defined, this behavior is modeled by an extra 1-D spatial discretization of the voltage $U_{r}$, meaning that $U_{r}=f(r)$, where continuous polynomial variations are possible approximations [4]. The total voltage $U$ across the foil winding in the $r$-direction is

$$
U=\frac{N_{f}}{h_{r}} \int_{h_{r}} U_{r} d r .
$$




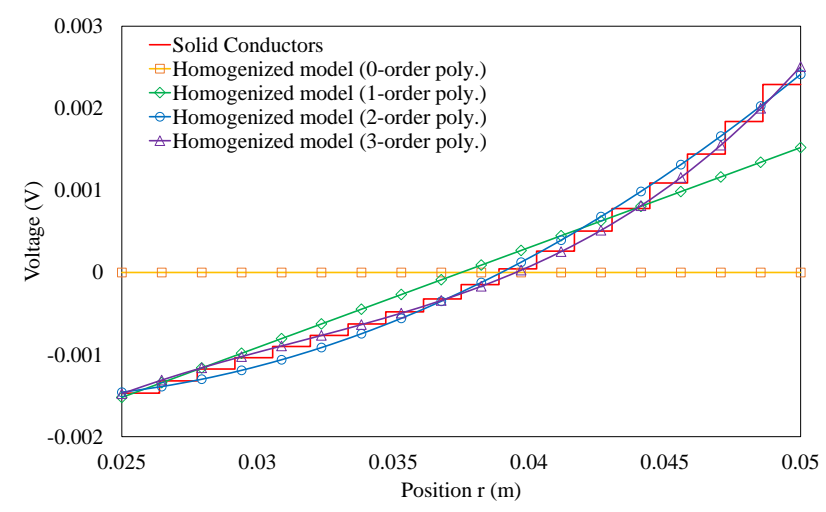

Fig. 2: Interturn voltage of the foil winding for a given instant of time $(t=0.17 \mathrm{~s})$.

Applying the Galerkin method, the magnetodynamic axisymmetric formulation is obtained from the weak form of the Ampere equation together with (3) as

$$
\begin{array}{r}
\int_{\Gamma} \frac{2 \pi}{r} \nu \operatorname{grad} \mathrm{a}_{\phi}^{*} \cdot \operatorname{grad} \mathrm{a}_{\phi}^{*^{\prime}} d \Gamma+\lambda \frac{\partial}{\partial t} \int_{\Gamma_{c}} \frac{2 \pi}{r} \sigma \mathrm{a}_{\phi}^{*} \mathrm{a}_{\phi}^{*^{\prime}} d \Gamma_{c} \\
+\lambda \int_{\Gamma_{c}} \frac{\sigma}{r} \mathrm{a}_{\phi}^{*^{\prime}} U_{r} d \Gamma_{c}=0, \quad \forall \mathrm{a}_{\phi}^{*^{\prime}}
\end{array}
$$

where $\nu$ is the reluctivity, $\mathrm{a}_{\phi}^{*}=r \mathrm{a}_{\phi}$ and the apostrophe differentiates test functions from basis functions. Likewise, the circuit relation from (4) can be written as

$$
\begin{array}{r}
\lambda \frac{\partial}{\partial t} \int_{\Gamma_{c}} \frac{2 \pi}{r} \sigma \mathrm{a}_{\phi}^{*} U_{r}^{\prime} d \Gamma_{c}+\lambda \int_{\Gamma_{c}} \frac{\sigma}{r} U_{r} U_{r}^{\prime} d \Gamma_{c} \\
\frac{N_{f} I_{f}}{h_{r}} \int_{\Gamma_{c}} U_{r}^{\prime} d r=0 .
\end{array}
$$

The equations to be solved for a voltage-driven foil winding system are (6) and (7) together with (5). Time derivates are approximated by the implicit Euler method.

\section{APPLICATION}

The proposed method is applied to a 2-D FE model of an axisymmetrical 18-turn foil winding inductor shown in Fig. 11b. A source of $2 \mathrm{~V}$ and $50 \mathrm{~Hz}$ is applied to the winding. The relative magnetic permeability of the core is $\mu_{r}$ core $=10$ and the electric conductivity of the foils is $59 \mathrm{MS} / \mathrm{m}$. A magnetic shielding box covers the core and winding (normal magnetic flux boundary condition on its inner surface) [4]. For simplicity, a unit fill factor is considered for the winding. The results are compared to those obtained by a FE model with all foils explicitly defined and represented as solid conductors.

The interturn voltage approximation is validated with different polynomial orders, Fig. 2 points out the accuracy of the homogenized method for a given time. In addition, the current density distribution along the axial direction for the first turn is presented in Fig. 3. The distribution depicts a concentration on the tips of the foil, explained by the absence of skin effect in the horizontal direction; similar behaviors are obtained for the remaining turns. The joule losses over time are also extracted and presented in Fig. 4. Transient phenomena is very well represented by the polynomial approximation. Only second

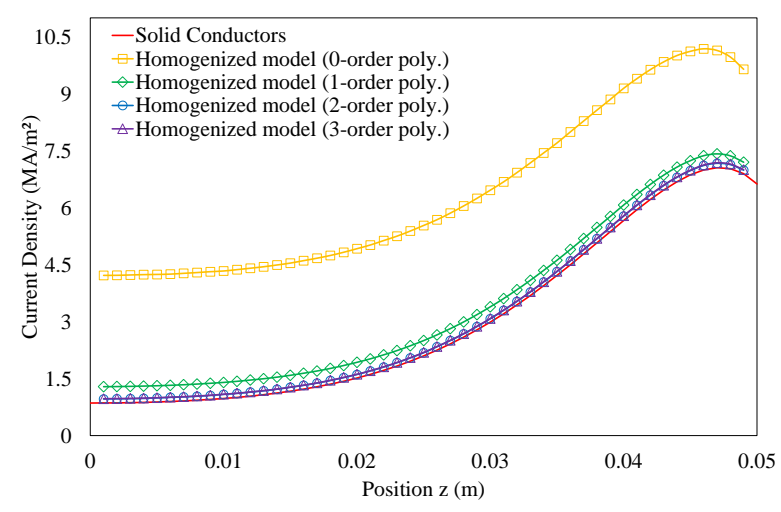

Fig. 3: Current density along half-height of the first turn (turn $1 / 18)$ for a given instant of time $(t=0.17 \mathrm{~s})$.

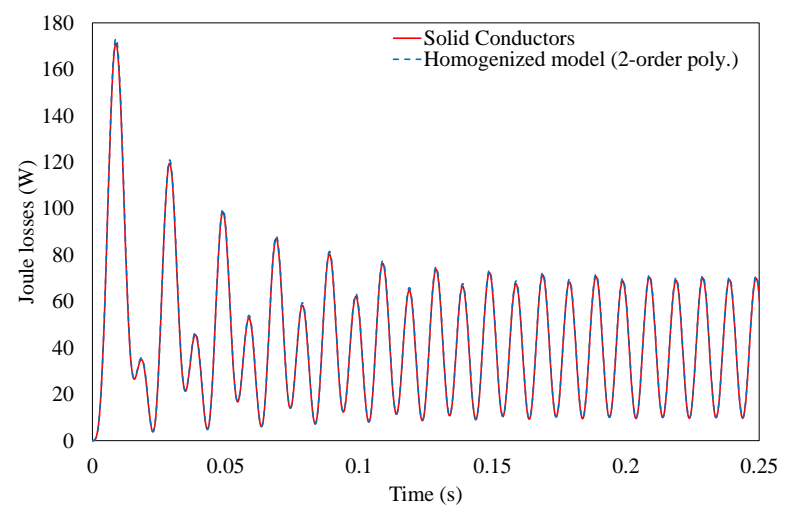

Fig. 4: Joule losses over time of the foil winding.

order polynomials are compared given their good performance with the interturn voltage representation.

\section{CONCLUSIONS}

A method has been proposed for time-domain foil-winding models. It allows to solve the eddy-current problem in a 2D axisymmetric FE model with good accuracy and reasonable computational cost. The method can be straightforwardly extended to 3-D FE models. In the extended paper, higher frequencies and the effect of the fill factor will be studied.

\section{REFERENCES}

[1] R. P. Wojda and M. K. Kazimierczuk, "Magnetic Field Distribution and Analytical Optimization of Foil Windings Conducting Sinusoidal Current," IEEE Magnetics Letters, vol. 4, pp. 0500204-0500204, 2013.

[2] G. Diaz and E. Mombello, "Semianalytic Integral Method for Fast Solution of Current Distribution in Foil Winding Transformers," IEEE Trans. Magn., vol. 51, no. 9, pp. 1-9, 2015.

[3] M. T. Villen, J. Letosa, A. Nogues and I. Murillo, "Procedure to Accelerate Calculations of Additional Losses in Transformer Foil Windings," Electric Power Systems Research, vol. 95, pp. 85-89, 2013.

[4] P. Dular and C. Geuzaine, "Spatially Dependent Global Quantities Associated With 2-D and 3-D Magnetic Vector Potential Formulations for Foil Winding Modeling," IEEE Trans. Magn., vol. 38, no. 2, pp. 633-636, March 2002.

[5] H. D. Gersem and K. Hameyer, "A Finite Element Model for Foil Winding Simulation," IEEE Trans. Magn., vol. 37, pp. 3427-3432, Sept. 2001. 